\title{
Hacer visible la reflexión docente: experiencia formativa innovadora en la asignatura de Tecnología Educativa II
}

\author{
Melissa Rodríguez Aguilar \\ lourdes.rodriguez@unah.edu.hn \\ Departamento de Pedagogía \\ Universidad Nacional Autónoma de Honduras
}

\section{Resumen}

1 presente artículo
analiza la experien-
cia formativa innova-
dora desarrollada en el primer período académico 2016, en la asignatura PA-209 Tecnología Educativa II, modalidad presencial, sección 1400,

12 de la Carrera de Pedagogía y 2016 Ciencias de la Educación, de la Universidad Nacional Autónoma de Honduras, UNAH, campus de Ciudad Universitaria.

La reflexión se hace a partir de la mirada del docente, y es lograda mediante la documentación de procesos, estrategias de enseñanza, vivencias y visiones que posibilitan la comprensión y transformación de las prácticas pedagógicas.

Ser observador crítico de esta práctica formativa, permitió profundizar en las motivaciones intrínsecas que promovieron los cambios, así como indagar sobre cuáles fueron los aprendizajes significativos que adquirieron los estudiantes en la asignatura de Tecnología
Educativa II. Se concluye con las lecciones aprendidas, desafíos y retos por enfrentar desde el conocimiento pedagógico, disciplinar y tecnológico con los cuales se construye la labor docente en el nivel superior.

\section{Palabras claves:}

Documentación de prácticas educativas, Docencia, Experiencias formativas, Innovación educativa.

\section{Abstract:}

The present article analyzes the formative and innovative experience developed in the first academic period of 2016, in the subject PA-209 Educative technologic II, presential model, section 1400, from the career of Pedagogy and Educational sciences. In the University National Autonomous from Honduras campus of University city. (UNAH-CU). This analyze could be from the teacher view, and is propitiate by trees fields, and teaching strategies, living and visions than comprehension and to transform academics practices.
To be the critical observer of this formative practice, it allows me to penetrate into the intrinsic motivations that promoted the changes, as well as to investigate on which they were the significant learnings that the students acquired in the subject of Educational Technology II. I conclude with the learned lessons, challenges and challenges for facing from the pedagogic knowledge, disciplining and technologically with which the educational labor is constructed in the top level.

Keywords:

Documentation of educational practices, Teaching, Formative experiences,

Educational innovation.

\section{Introducción}

“'Mi práctica pedagógica ha sido la más adecuada?” Es una de las preguntas que realizan la mayoría de los docentes, en el desarrollo de las asignaturas a nivel superior. Las inquietudes son muchas, y las respuestas muy variadas. Se 
recomienda para ello utilizar la documentación de experiencias didácticas como método de investigación, para reconocer aciertos y errores, que promuevan reflexiones encaminadas a mejorar el proceso enseñanza-aprendizaje.

Es en este contexto se sitúa el análisis de la práctica docente e incorporación de innovaciones en la asignatura de Tecnología Educativa II, generadas a raíz de la necesidad de establecer nuevas propuestas formativas que den respuesta al abordaje de contenidos curriculares que respondan a la exigencia de la formación de profesionales de la pedagogía con conocimientos, habilidades y actitudes para el desarrollo de la educación virtual.

Para ello se estableció un marco referencial en torno a las tendencias internacionales del perfil del pedagogo y se concluye que los estudiantes deben cursar la asignatura de Tecnología Educativa II con un enfoque más actualizado, dirigido al e-learning y al diseño, ejecución y evaluación de procesos formativos virtuales.

Es así como se diseña e implementa la experiencia innovadora: Creación de cursos virtuales para estudiantes de PA-101 Pedagogía General, cuyo proceso de desarrollo parte de un análisis previo que incluye la justificación y contextualización para después dar cabida al análisis de la dimensión educativa, pedagógica y didáctica propuestas por Jonassen, (2000) y Lefoe, (1998) citados por Tenorio, L., \& Taquez, H. (2016).

Se finaliza el artículo con un marco de conclusiones que permiten evidenciar las buenas prácticas, así como los desaciertos, con el fin de reconstruir nuevas propuestas formativas a partir de la documentación de experiencias innovadoras por medio de TIC que visibilizan la acción del docente y convierten el conocimiento pedagógico en algo explícito.

Justificación de la experiencia formativa innovadora

Según María Libedinsky (2015) la innovación didáctica hace alusión a dos componentes: rupturas y continuidades. ¿Cuáles son, entonces, las rupturas y continuidades que deben darse en la asignatura de Tecnología Educativa II?

Una mirada a la situación actual de esta asignatura, nos indica que la materia de PA209 Tecnología Educativa II, cuenta con tres unidades valorativas U.V (se refiere a tres horas de clases a la semana), pertenece al sexto período del plan de estudios vigente (1994) de la licenciatura en Pedagogía con orientación de Planeamiento y Administración de la Educación.

Como la motivación principal era la actualización curricular, el trabajo se inició con la revisión de los objetivos educacionales de la asignatura en mención. En esa línea se identificó la primera ruptura: dejar aún lado algunos objetivos del plan de estudio vigente, puntualmente el relacionado a manejar criterios teóricos y prácticos para el análisis y diseño de mensajes educativos, los mismos sistemas de información automatizados.

La segunda decisión consistió en darle continuidad, aunque con otra perspectiva a los objetivos siguientes: Analizar el modelo de comunicación alternativa como promotora de un proceso efectivo de instrucción. Capacitar en el manejo de la terminología básica de la comunicación y en la elaboración de diseños instruccionales.

Después de definir los objetivos, $\frac{13}{2016}$ se pasó a identificar las actividades pedagógicas por desarrollar, consideramos lo siguiente: "las acciones innovadoras han de focalizarse en el programa formativo del aula (currículum) y valorar su pertinencia para la educación integral de los estudiantes en el marco de la sociedad del conocimiento, los retos interculturales y los proyectos y acciones sociolaborales". (Domínguez, Medina, y Sánchez, 2011, pág. 66).

El repensar el ambiente de aprendizaje implicó preguntarse: ¿qué estoy enseñando?, ¿cómo lo estoy enseñando? y ¿para qué lo estoy enseñando? Es por lo que el ambiente 
de aprendizaje es entendido como aquel espacio construido simbólicamente por el docente, para alcanzar los objetivos deseados y que implica un proceso de reflexión constante de la tarea formativa.

En esta reflexión también se debe pensar críticamente sobre el rol mediador de la incorporación de las TIC en los ambientes de aprendizaje universitarios, lo cual supone un proceso complejo.

Diseñar aulas virtuales mediante cualquier tipo de plataformas, o realizar investigaciones en temas vinculantes a las Tecnologías y educación no implica un proceso innovador por sí solo. Salinas (2004) señala que el desafío que deben de emprender las universidades radica en "revisar sus referentes actuales y promover experiencias innovadoras en los procesos de enseñanza-aprendizaje, apoyándose en las TIC y haciendo énfasis en la docencia, en los cambios de estrategias didácticas de los profesores y en los sistemas de comunicación y distribución de los materiales de aprendizaje" (pág. 2). Es decir, lo importante siempre será poner la atención en lo pedagógico, y luego en lo tecnológico.

En relación con lo anterior, la experiencia formativa de la asignatura de Tecnología Educativa II, se desarrolló siguiendo las características y bases del $e$-learning, partiendo de que se entiende como "un proceso de formación a distancia (reglada o no reglada), basado en el uso de las tecnologías de la información y las telecomunicaciones, que posibilitan un aprendizaje interactivo, flexible y accesible, a cualquier receptor potencial" (Cabero, 2006, pág. 2).

Este tipo de formación basada en la red permite la combinación de diversos materiales (auditivos, visuales y audiovisual), proporcionados intencionalmente por el docente. Asimismo, el conocimiento se concibe como un proceso activo de construcción.

En esta línea de reconocer el impacto actual y expansión de los espacios formativos utilizando el potencial de las TIC, el Informe Horizontes (2016, pág. 16) plantea una tendencia en la adopción de nuevas tecnologías en la educación superior en los próximos uno a dos años, es decir a corto plazo.

El informe señala el uso generalizado de sistemas de gestión de aprendizaje (LMS), incluidos la Blackboard y Moodle, "que almacenan grandes cantidades de datos relacionados con las actividades de los estudiantes" (pág. 16).

Esto abre la posibilidad de construir ambientes de aprendizajes innovadores y evidencia un gran desafío para los docentes, principalmente para actualizarse y capacitarse en plataformas flexibles y de fácil funcionamiento como la Moodle.
Actualmente Moodle es la plataforma de soporte tecnológico del modelo de educación virtual de la UNAH. Esta reúne las condiciones para un entorno virtual de formación que posibilite experiencias de aprendizaje al estudiante, se articula en torno a tres módulos: comunicación, materiales y actividades (Llorente, 2006).

Fundamento del análisis de la experiencia formativa innovadora en la asignatura de Tecnología Educativa II La configuración y sistematización de esta experiencia formativa se fundamenta en los planteamientos y aportes de Jonassen, (2000), Lefoe, (1998) citados por Tenorio, L., \& Taquez, H. (2016) y Libedinsky (2001).

\section{Dimensión educativa:}

Para esta dimensión se analizó el contexto, antecedentes y la problemática educativa que motivó a realizar una experiencia formativa innovadora. Como resultado de ello se identificó la descontextualización de los objetivos, contenidos, estrategias didácticas del programa de la asignatura Tecnología Educativa II. Los resultados del Informe de Autoevaluación Fase cualitativa de la Carrera de Pedagogía (2012), evidencian que los estudiantes carecen de habilidades en el uso y manejo de TIC, situación que demanda rupturas que modifiquen e innoven la manera en que se ha desarrollado a nivel teórico y práctico la asignatura de Tecno- 
logía Educativa I, siempre bajo la mirada integral de lo que debe ser la formación del profesional de la pedagogía del siglo XXI.

Las tendencias internacionales en cuanto a la formación del pedagogo, según el proyecto $\mathrm{Tu}-$ ning para América Latina (2013) señalan que las competencias específicas en el área de educación son: Selecciona, utiliza y evalúa las tecnologías de la comunicación e información como recurso de enseñanza y aprendizaje. Produce materiales educativos acordes a diferentes contextos para favorecer los procesos de enseñanza y aprendizaje.

Estas nuevas tendencias del mundo universitario de hoy abren desde luego otros escenarios laborales para los profesionales de la pedagogía, a la vez que les exige nuevas competencias para desempeñarse $y$ acompañar procesos educativos innovadores, donde las TIC están presentes como herramientas didácticas mediadoras.

No hay discusión respecto a que los educadores de hoy necesitan adquirir capacidades, habilidades y actitudes pertinentes para el aprendizaje mediado por TIC.

\section{Dimensión pedagógica}

Esta dimensión es abordada desde el qué y para qué, es decir cuáles son los aprendizajes que se promueven en la experiencia formativa y reflejar en qué medida las TIC contribuyeron para dichos aprendizajes.

Los objetivos principales estuvieron enfocados a:

- Reflexionar críticamente sobre la cultura digital y los procesos pedagógicos emergentes mediados por las TIC.

- Utilizar diversas herramientas informáticas para el trabajo colaborativo.

- Fundamentar didácticamente propuestas formativas que incorporen estrategias y medios o materiales educativos en contextos de la enseñanza a distancia (con énfasis en la virtualidad).

- La adquisición de conocimientos, habilidades y actitudes que les posibilite ser asesores/tutores en espacios formativos virtuales.

Además de los objetivos anteriores, los estudiantes debían tener un acercamiento genuino y experimentar lo que es ser un estudiante en la virtualidad. Por ello, la experiencia formativa se planificó en un ambiente de aprendizaje b-learning.

Según Cabero (2006) algunas de las muchas ventajas del aprendizaje en línea están relacionadas con la autonomía del estudiante, el uso de las diferentes herramientas de comunicación sincrónica y asincrónica para el estudiante y el profesor, además, permite que en los servidores puedan quedar registradas las actividades realizadas por los estudiantes.

En consecuencia, se inició con la creación de un aula virtual ${ }^{1}$ en la plataforma del campus virtual de la UNAH, que siga una organización metodológica, orientada por los objetivos de la clase (Ver tabla 1).

Los estudiantes asistían a sus clases presenciales de lunes a miércoles como lo indica el plan de estudio, y accedían al espacio de aprendizaje en línea en el horario flexible que ellos determinaban de acuerdo con su conveniencia.

Las unidades didácticas desarrolladas fueron:

Unidad I. Delimitación del campo de acción de la Tecnología Educativa

Unidad II. Diseño de propuestas formativas con el uso de las TIC

Unidad II: Los actores del proceso de enseñanza y aprendizaje virtual

\section{Dimensión didáctica}

En esta dimensión se analizan las fases y estrategias didácticas desarrolladas en la asignatura.

\section{Fase I: De estudiantes pre- senciales a estudiantes vir- tuales \\ Como se indicó en párrafos an-}

\footnotetext{
${ }^{1 .}$ La Dirección de Innovación Educativa DIE, promueve cursos y capacitaciones para el diseño de las aulas virtuales en la Plataforma de la UNAH, de la cual he sido beneficiada, y ha contribuido a fortalecer mis capacidades y así poder llevar a cabo esta experiencia formativa.
} 
Tabla 1. Organización metodológica del aula virtual para la asignatura de PA- 209-Tecnología Educativa II

\begin{tabular}{|c|c|c|}
\hline $\begin{array}{c}\text { Actividades de enseñanza y } \\
\text { aprendizaje virtual }\end{array}$ & $\begin{array}{c}\text { Funciones } \\
\text { ¿Para qué deseo hacer la } \\
\text { incorporación virtual? }\end{array}$ & $\begin{array}{c}\text { Recursos utilizados del } \\
\text { Campus Virtual (Plataforma } \\
\text { Moodle) * }\end{array}$ \\
\hline \multirow{5}{*}{$\begin{array}{l}\text { - Las comunidades virtuales de } \\
\text { aprendizaje. } \\
\text { - El aprendizaje basado en el uso } \\
\text { autónomo de recursos digitales } \\
\text { telemáticos. } \\
\text { - La búsqueda de información en } \\
\text { Internet. } \\
\text { - Las discusiones virtuales. } \\
\text { - El trabajo cooperativo virtual. } \\
\text { - Las actividades de autoevalua- } \\
\text { ción } \\
\text { - La producción de presentaciones } \\
\text { multimedia }\end{array}$} & Responsabilizadora & $\begin{array}{l}\text {-Glosario } \\
\text {-Carpetas } \\
\text {-Lecturas obligatorias } \\
\text {-Actividades subir archivo. } \\
\text {-URL }\end{array}$ \\
\hline & Socializadora & $\begin{array}{l}\text {-Foros } \\
\text {-Subir archivo }\end{array}$ \\
\hline & Comunicativa & $\begin{array}{l}\text {-Foros de reflexión } \\
\text {-Autoevaluaciones } \\
\text {-Juegos: Crucigrama }\end{array}$ \\
\hline & Motivadora & $\begin{array}{l}\text {-Foros de reflexión } \\
\text {-Foros debate }\end{array}$ \\
\hline & Evaluadora & $\begin{array}{l}\text {-Cuestionarios } \\
\text {-Subir archivo }\end{array}$ \\
\hline Fuente: Elaboraci & $\begin{array}{l}\text { los recursos: https://docs.moodl } \\
\text { n propia a partir de (Barbera y Ba }\end{array}$ & $\begin{array}{l}\text { org/all/es/Recursos } \\
\text { ia, 2005). }\end{array}$ \\
\hline
\end{tabular}

16

2016 teriores, los estudiantes de Tecnología Educativa II no podrían desarrollar propuestas formativas virtuales para otros estudiantes si previo, ellos no experimentaban lo que significa ser un estudiante virtual. Por ello se diseñaron estrategias pedagógicas dentro del aula virtual de la asignatura a fin de promover el trabajo autónomo y colaborativo, así como el uso de recursos como: el Cmpatools, Edraw Mind Map, Google Docs, entre otros, que facilitan el e-learning.

\section{Fase II: El diagnóstico}

En esta fase se decidió que los estudiantes que recibirían los cursos virtuales que se diseñaran y desarrollaran en Tecnología Educativa II, serían los alumnos de la asignatura PA-
101 Pedagogía General, sección 1600 , del primer periodo académico, 2016.

Para ello se elaboró un instrumento que logró identificar las necesidades de formación en las siguientes áreas: a) Estrategias de aprendizaje. b) Hábitos de estudio. c) $\mathrm{Ma}-$ nejo del Programa Microsoft Office. d) Estrategias para hablar en público. e) Seguridad informática. Además, dicho diagnóstico posibilitó conocer las características de los beneficiarios de los cursos, elemento esencial para todo proceso formativo.

Fase II: Construcción colectiva de cursos virtuales

Esta es quizás, la fase más compleja que tuvieron que asumir los estudiantes de Tecnología Educativa II. Hubo dos momentos, el de diseño, y el de ejecución.

-Diseño: en esta fase se conformaron cinco grupos de trabajos por afinidad. El diseño de los cursos virtuales tuvo como sustento teórico la planificación didáctica y diseño instruccional en ambientes virtuales (Chacín, 2011; Sandoval, 2010). Es así como los estudiantes diseñaron actividades, organizaron los materiales y recursos didácticos pensando en las características y necesidades de los beneficiarios de los cursos.

Otro de los aspectos desafiantes para los estudiantes de Tecnología Educativa II en esta 
Tabla 2. Distribución de los estudiantes según los cursos virtuales

\begin{tabular}{lcc}
\hline \multicolumn{1}{c}{ Nombre de los cursos virtuales } & $\begin{array}{c}\text { Estudiantes matriculados } \\
\text { de Pedagogía General } \\
\text { (Beneficiarios) }\end{array}$ & $\begin{array}{c}\text { de Tecnología } \\
\text { Educativa II } \\
\text { (Diseñadores/ } \\
\text { administradores } \\
\text { yasesores de los } \\
\text { cursos) }\end{array}$ \\
\hline $\begin{array}{l}\text { Estrategias para realizar una excelente } \\
\text { exposición }\end{array}$ & 10 & 5 \\
\hline $\begin{array}{l}\text { El buen uso de programas Microsoft } \\
\text { Office }\end{array}$ & 9 & 5 \\
\hline Cuidándonos en el Ciberespacio & 8 & 5 \\
\hline $\begin{array}{l}\text { Aprende, comprende y práctica las } \\
\text { técnicas didácticas }\end{array}$ & 8 & 6 \\
\hline Aprender haciendo & 8 & 6 \\
\hline Total & 43 & 27 \\
\hline
\end{tabular}

fase, fue el manejo de la plataforma Moodle. En consecuencia, se planificaron cinco talleres, acompañados de recursos que facilitarán la comprensión de la plataforma. La Dirección Ejecutiva de Gestión de Tecnológica, DEGT, les proporcionó acceso para ser administradores del aula virtual de cada curso. Las jornadas de trabajo se desarrollaron en la sala de computación de la $\mathrm{Ca}-$ rrera de pedagogía. Disponer de ese espacio de capacitación tecnológica se constituyó en una ventaja.

En todo el proceso fue necesario la revisión y retroalimentación de los guiones instruccionales por la docente de Tecnología Educativa II. Ese proceso contribuyó a que las tres unidades didácticas de cada curso virtual cumplieran con los requerimientos pedagógicos y tecnológicos para la calidad de estos.

Seguidamente, se planificó una jornada de inducción al Campus Virtual y presentación de los cursos virtuales a los estudiantes de Pedagogía General, previamente matriculados en los cursos según su preferencia. En dicha jornada se explicaron los propósitos y metodología a seguir en cada curso virtual (Ver tabla 2).

\section{La implementación de los cursos virtuales}

El período de ejecución fue del 18 de abril al 6 de mayo, al finalizar el primer período académico del 2016. En esta fase los estudiantes de Tecnología Educativa II desarrollaron habilidades de asesor en línea, aplicando conocimientos sobre seguimiento y acompañamiento a los estudiantes inscritos en cada curso en línea. Esto les exigió el desarrollo de competencias para facilitar y dinamizar el proceso enseñanza y aprendizaje en línea, motivar la participación e interacción de los alumnos de los cursos virtuales. De igual forma evaluar el proceso identificando posibilidades y limitaciones de los espacios de aprendizaje virtuales generados en función de los objetivos didácticos.

\section{Fase VI}

La evaluación de toda la experiencia formativa innovadora: aciertos y desafíos

Como producto educativo final, los estudiantes de Tecnología Educativa II elaboraron un informe donde sistemati- 


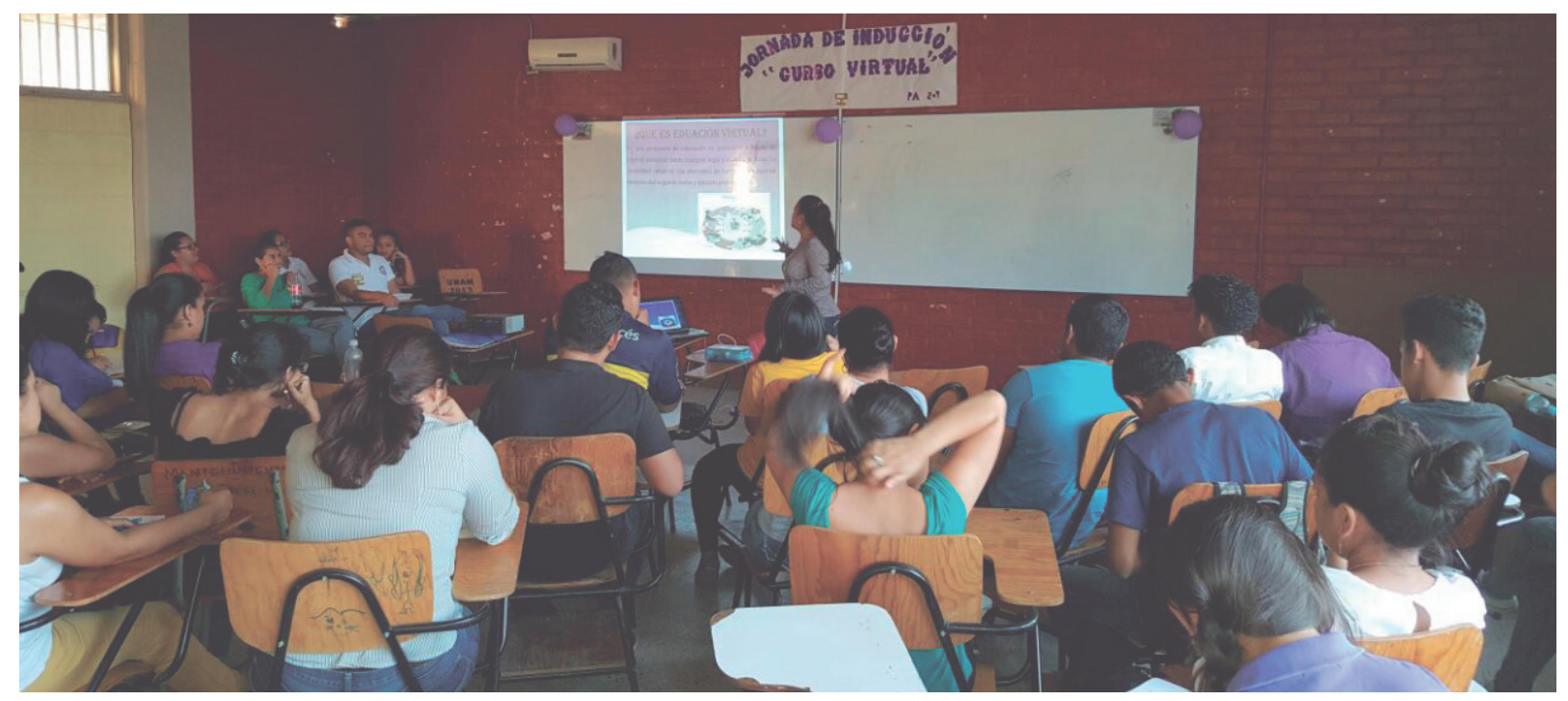

Estudiantes de PA-209 Tecnología Educativa II, brindando el curso de inducción a los estudiantes de Pedagogía General.

zaron sus experiencias, plasmaron la experiencia formativa, incluyeron la evaluación de cada uno de los cursos desarrollados e implementados.

\section{8}

El proceso de evaluación implicó la aplicación de entrevistas y cuestionarios que recogieron el sentir y pensar de los estudiantes de los cursos virtuales impartidos.

Como parte de los resultados de las evaluaciones, se evidenció que "un $90 \%$ de los estudiantes les pareció muy interesante y entretenido cada uno de los contenidos y las actividades programadas, y a un $10 \%$ no les gustó mucho ya que expresaron que les hubiesen gustado más contenidos y actividades". (Mejía, y otros, 2016).

En el mismo orden de ideas, "otro aprendizaje significativo fue que nunca había cursado un curso en línea y fue muy interesante la actividad y así cuando quiera llevar una clase en línea ya tengo los conocimientos necesarios sobre cómo utilizar esta plataforma". (Sevilla, y otros, 2016).

\section{Reflexiones finales}

La documentación de esta experiencia formativa innovadora con TIC permitió al docente posicionarse en su práctica pedagógica, y concebir su quehacer educativo como un hecho reflexivo, colectivo y visible para la construcción de conocimiento disciplinar, pedagógico, didáctico y tecnológico, acorde a una realidad educativa cambiante.

La innovación implica ante todo una praxis que promueve rupturas conceptuales, formas de percibir y evaluar la realidad, para ello el docente debe realizar una reflexión crítica continua sobre: ¿qué enseño? ¿para qué enseño? ¿cómo enseño? Los resultados muestran que las propuestas educativas me- diadas por las TIC son cada vez más sólidas y aceptadas por la comunidad estudiantil. Para los estudiantes de Tecnología Educativa II y de Pedagogía General los espacios formativos en línea desarrollados y puestos en práctica se convirtieron en escenarios de aprendizaje dinámico e innovadores tanto a nivel de contenidos como de actividades didácticas. Sin embargo, demandan del estudiantado capacidades tecnológicas, no poseerlas se convierte en una gran limitante, tanto para el que diseña cursos virtuales mediante la plataforma Moodle, como para los que se inscriben en estos cursos en línea.

Pero, más allá del manejo tecnológico de las aulas virtuales, asimilar el aprendizaje en línea o e-learning implica adquirir una serie de capacidades y actitudes pedagógicas y didácticas que posibiliten el aprendizaje 
autónomo, la autorregulación, la ética a fin de aprovechar las potencialidades educativas de las TIC para el aprendizaje a lo largo de la vida. El desarrollo de estas competencias deberá ser parte de los currículos académicos.

\section{Referencias}

Cabero, J. (2006). Bases pedagógicas del e-learning. Revista de Univesidad y Sociedad del Conocimiento, 3(1), 1-10.

Chacín, A. (2011). La planficación didáctica y el diseño instruccional en ambientes virtuales. Redalyc, 26(2), 129-160.

Dominguez Garrido, M. C., Medina Rivilla, A., \& Sánchez Romero, C. (2011). La Innovación en el aula: referente para el diseño y desarrollo curricular. Redalyc, 61-86.

Domínguez, M. C., Medina, A., \& Sánchez, C. (2011). La innovación en el aula: referente para el diseño y desarrollo curricular. Perspectiva Educacional, 50(1)

Johnson, L., Adams Becker, S., Cummins, M., Estrada, V., Freeman, A., y Hall, C. (2016). NMC Informe Horizon 2016 Edición Superior de Educación. Austin, Texas: The New Media Consortium.

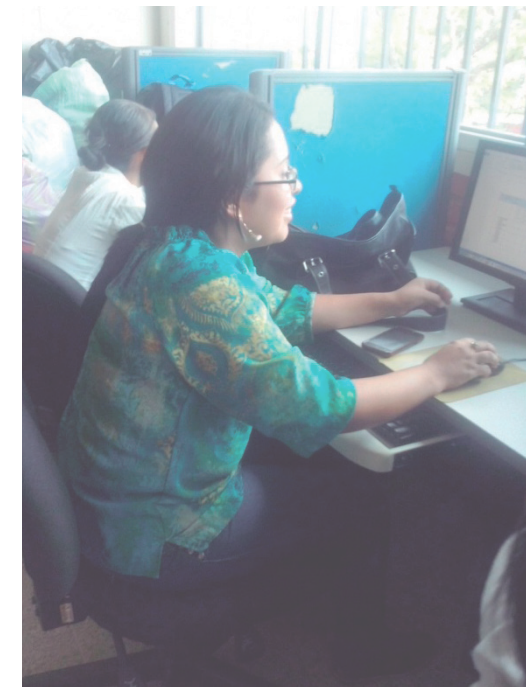

Estudiante de Tecnología Educativa II diseñando el curso virtual "Aprender Haciendo".

Libedinsky, M. (2001). La innovación en la enseñanza: Diseño y documentación de experiencias de aula. Buenos Aires: Paidós SAICF.

Libedinsky, M. (2015). Congreso de Educación y Tecnología de la Provincia de Santa Cruz. Recuperado el Agosto de 2016, de Cómo documentar experiencias de enseñanza innovadoras con TIC.

Lefeo, G. (1998). Creating constructivist learning envronments on the web: The challenge in higher education. Paper presented at the ASCILETE, 1998, Wollongong, Australia. 453-464

Llorente, C. (2006). Moodle como entorno virtual de formación al alcance de todos. Redalyc, XV(28), 197202.
Mejia, L., Lagos, W., Matamoros, R., Lemus, M. E' Herrera, M. (2016).Informe final, grupo 3. Trabajo presentado en la asignatura de Tecnología Educativa II, Carrera de Pedagogía. Honduras: UNAH.

Salinas, J. (2004). Innovación docente y uso de las TIC en la enseñanza universitaria. Revista Universidad y Sociedad del Conocimiento, 1(1), 2.

Sandoval , C. (2010). Dimensión Pedagógica de la Educación Virtual: Una reflexión pendiente. Pedagogía $y$ Saberes(32), 33-44.

Sevilla, K., Rivas, A., Salinas, A. \& Martínez, R. (2016). Informe final, grupo 2. Trabajo presentado en la asignatura de Tecnología Educativa II, Carrera de Pedagogía. Honduras: UNAH.

Tenorio, L., \& Taquez, H. (2016). Ambientes de aprendizaje mediados por las TIC: Encontrando sentido en la escuela. Recuperado el 18 de agosto de 2016, de eduteka: http://eduteka.icesi.edu.co/ articulos/aprendizaje-mediado-por-tic

Tuning. (2013). Tuning para América Lanina. Recuperado el Febrero de 2016, de http://www.tuningal.org/

Departamento de Pedagogía. (2012). Informe de Autoevaluación-Fase Cualitativa de la Carrera de Pedagogía. Honduras: UNAH 